\title{
INTERFEROMETRY ON MIRA IN THE MID-INFRARED: CYCLIC VARIABILITY OF THE CONTINUUM DIAMETER AND THE EFFECT OF SPECTRAL LINES ON APPARENT SIZE
}

\author{
J. Weiner, D. D. S. Hale, and C. H. Townes \\ Space Sciences Laboratory and Department of Physics, University of California, Berkeley, CA 94720; johnw@ssl.berkeley.edu, \\ david@isi.mtwilson.edu,cht@ssl.berkeley.edu \\ Received 2002 October 24; accepted 2003 January 21
}

\begin{abstract}
The size and variability of the continuum photosphere of $o$ Ceti have been measured with $11 \mu \mathrm{m}$ heterodyne interferometry to an accuracy of about $1 \%$. Narrow bandwidths $\left(\sim 0.17 \mathrm{~cm}^{-1}\right)$ were used to avoid spectral lines and measure continuum only. The resulting $11 \mu \mathrm{m}$ diameter of $o$ Cet is larger than the previously measured visible and near-infrared sizes. In addition, variations in the diameter with phase and a possible elongation have been observed. Visibilities were also measured at wavelengths known to contain strong $\mathrm{H}_{2} \mathrm{O}$ spectral contamination, giving larger apparent stellar sizes and information on the distribution of hot $\mathrm{H}_{2} \mathrm{O}$ gas.
\end{abstract}

Subject headings: infrared: stars — stars: AGB and post-AGB — stars: atmospheres stars: individual (o Ceti) - techniques: interferometric

\section{INTRODUCTION}

Mira variables are extremely large and cool asymptotic giant branch stars that pulsate more or less regularly. Their visual brightness can vary by as much as 8 mag, with a period on the order of a year. Pulsationally induced shock waves, formed in the lower atmosphere, significantly affect the density and temperature structure of the star. The stellar "surface" becomes extended and obscured by dust and molecular opacity of the atmosphere.

Measurements of the angular diameters of Mira variables provide a direct way to test and differentiate between the dynamical models constructed to understand Mira structure. In particular, simultaneous measurements at multiple wavelengths probe different layers of the stellar atmosphere (because of the varying opacity), and measurements of the changes in size over the course of a cycle provide a direct measure of the pulsation occurring.

Within the past two decades, an impressive quantity of stellar diameters have been measured. Published size measurements of $o$ Cet are listed in Table 2. There are large discrepancies between some of these values at different wavelengths. Variations in the visible and near-infrared measurements of up to a factor of 2 are present. The wavelength-dependent size fluctuations evident in Table 2 illustrate the importance of the interpretation of diameter measurements for large, extended asymptotic giant branch (AGB) stars such as Mira. These stars are not adequately described by compact atmosphere models. Still more important is the opacity arising from dust and molecular transitions in the atmosphere that can, at some wavelengths, induce large variations in the apparent size of the star. To further complicate matters, asymmetries (in some cases, time-dependent asymmetries) have also been observed in $o$ Cet in the visible by Karovska (1997), Wilson et al. (1992), Tuthill, Monnier, \& Danchi (1999b), and Josselin et al. (2000). These may result from the excitation of nonradial modes of pulsation or from hot spots caused by convective cells. ${ }^{1}$ The hot spots furthermore may be exaggerated in the

\footnotetext{
${ }^{1}$ Schwarzschild (1975) argues that convective granules, observed on the solar surface to have typical size $0.003 R_{\odot}$, should exist on the surface of AGB stars having size on the order of the stellar radius. These could produce (asymmetric) hot spots having temperature $\sim 1000 \mathrm{~K}$ hotter than the surrounding surface.
}

intensity distribution by extremely temperature-dependent opacities such as TiO bands (Young et al. 2000). Such large hot spots may be the dominant feature of the star at visible wavelengths and are capable of lowering its apparent size significantly.

The mid-infrared is well suited for measuring diameters because of the relatively weak dependence of intensity on temperature and the lower scattering by dust particles known to inhabit the circumstellar regions as compared to shorter wavelengths. However, thermal emission from circumstellar dust is significant in the mid-infrared, and attempts must be made to separate the flux contribution of the dust shell from that of the stellar disk. Any hot spots or temperature inhomogeneities present in the photosphere would have a much weaker effect on the mid-infrared apparent size than at visible or near-infrared wavelengths.

The Infrared Spatial Interferometer (ISI) is particularly useful for studying angular diameters because its wavelength range is between 9.5 and $11.5 \mu \mathrm{m}$, and its narrow bandpass (having width $\sim 0.17 \mathrm{~cm}^{-1}$ in wavenumber) can either avoid or observe on spectral lines. For these observations, the ISI consisted of two telescopes with $1.65 \mathrm{~m}$ apertures separated by various baselines up to $56 \mathrm{~m}$. The telescopes employ heterodyne detection (with a $\mathrm{CO}_{2}$ laser in each telescope serving as the local oscillator) to measure visibilities. The ISI is located at Mount Wilson Observatory, chosen for its excellent seeing and relative lack of fast atmospheric turbulence. A complete description of the instrument can be found in Hale et al. (2000). A third telescope has recently been added to the system.

\section{OBSERVATIONS}

The stellar diameters obtained with the ISI are the first measurements of a stellar disk at wavelengths longer than about $4 \mu \mathrm{m} .{ }^{2}$ The diameter is obtained from the raw data (spatial frequency $\left[x_{i}\right]$, measured visibility squared $\left[V_{i}^{2}\right]$, and error $\left[\sigma_{V_{i}^{2}}\right]$ ) by fitting it with a uniform disk visibility curve. The raw visibilities were calibrated by observing $\alpha$ Tau,

\footnotetext{
2 There have been observations at radio wavelengths with resolutions high enough to resolve stars, but they do not actually " see" the stellar disk at these wavelengths. See Reid \& Menten (1997).
} 


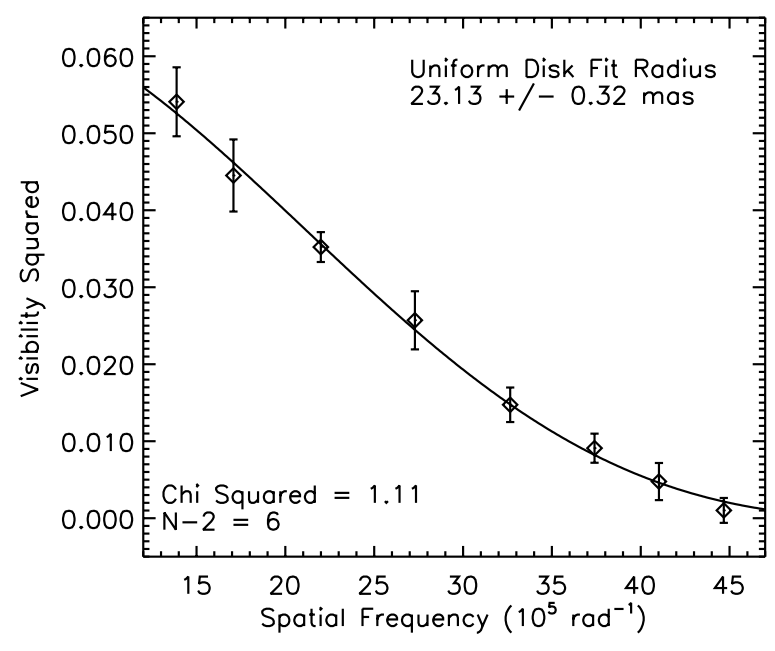

FIG. 1.-ISI Data for $o$ Cet from the night 2000 November 28 fitted with a uniform disk. The best-fit uniform disk has a radius of 23.13 mas, with $25.7 \%$ of the light coming from the stellar disk.

which was assumed to have a diameter of 22 mas and no significant circumstellar dust. The presence of dust at larger radii around $o$ Cet is allowed for with a second parameter in the fit, $A$, which lowers the visibilities by an amount equal to the fraction of light coming from the dust shell. The visibility function for the range of spatial frequencies measured is given by

$$
V(x, r, A)=\frac{2 A J_{1}(2 \pi r x)}{2 \pi r x},
$$

where $x$ is the spatial frequency in $\operatorname{rad}^{-1}, J_{1}$ is the Bessel function of order unity, and $r$ is the radius of the stellar disk in radians. The best-fit radius is given by the minimum of $\chi^{2}(r, A)$, where

$$
\chi^{2}(r, A)=\frac{\sum_{i=1}^{N}\left\{\left[V\left(x_{i}, r, A\right)\right]^{2}-V_{i}^{2}\right\}^{2}}{\sigma_{V_{i}^{2}}^{2}} .
$$

Any error in calibration of the visibility, provided it is constant throughout the observation, is absorbed into the parameter $A$ when fitted and does not affect the diameter measurement. The error in the best-fit stellar disk radius, $\sigma_{r}$, was estimated by considering the region of the $A-r$ plane in which the $\chi^{2}$, normalized such that its minimum value is $N-2$, is increased by no more than unity from this minimum. Here $\sigma_{r}$ was taken to be one-half the width of this region in $r$. This general procedure is outlined in Bevington (1969).

An example of a uniform disk fitted to a relatively good observing night's data on $o$ Cet is shown in Figure 1. Often, as in this case, a single night of observations yields enough data to be reasonably fitted with a uniform disk model. For weaker stars, or in worse seeing conditions, we may combine several nights together before fitting. There is rarely a case in which data are combined that span more than a week. It should be noted that the uniform disk diameter is essentially just a convenient parameter that characterizes the stellar size. In reality, the sharpness of the edge of the disk is a higher resolution feature that cannot be measured at the spatial frequencies observed.
TABLE 1

\begin{tabular}{|c|c|c|c|c|}
\hline Dates & Phase & $\begin{array}{c}\lambda \\
(\mu \mathrm{m})\end{array}$ & $A^{\mathrm{a}}$ & $\begin{array}{l}\text { Diameter } \\
\text { (mas) }\end{array}$ \\
\hline 1999 Oct $22,26 \ldots$ & 0.99 & 11.149 & 0.276 & $46.56 \pm 1.43$ \\
\hline 1999 Nov $10-19$.............. & 0.06 & 11.149 & 0.327 & $49.25 \pm 0.55$ \\
\hline 2000 Sep 30 , Oct 3-6..... & 0.03 & 11.149 & 0.310 & $47.63 \pm 0.80$ \\
\hline 2000 Oct $17-20 \ldots \ldots \ldots \ldots . .$. & 0.08 & 11.149 & 0.326 & $48.84 \pm 0.91$ \\
\hline 2000 Nov 1 ...................... & 0.12 & 11.149 & 0.345 & $48.25 \pm 0.94$ \\
\hline 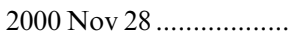 & 0.20 & 11.149 & 0.433 & $47.35 \pm 1.24$ \\
\hline 2000 Dec $17,21 \ldots \ldots \ldots \ldots . .$. & 0.27 & 11.149 & 0.465 & $46.48 \pm 0.84$ \\
\hline 2001 Jul 18-31 ............... & 0.92 & 11.149 & 0.456 & $50.67 \pm 0.63$ \\
\hline 2001 Aug 1-8 .................. & 0.95 & 10.884 & 0.392 & $57.90 \pm 1.52$ \\
\hline 2001 Aug $22,24 \ldots \ldots \ldots \ldots$ & 0.01 & 11.149 & 0.384 & $53.18 \pm 0.67$ \\
\hline 2001 Sep 25-27................ & 0.11 & 11.149 & 0.411 & $53.99 \pm 0.53$ \\
\hline 2001 Oct $4,5 \ldots \ldots \ldots \ldots \ldots$ & 0.13 & 11.149 & 0.348 & $51.44 \pm 0.67$ \\
\hline 2001 Oct 11 ....................... & 0.15 & 11.149 & 0.346 & $54.27 \pm 1.67$ \\
\hline 2001 Oct $23,24 \ldots \ldots \ldots \ldots \ldots$ & 0.19 & 11.149 & 0.366 & $55.06 \pm 0.58$ \\
\hline 2001 Oct 25,26, Nov $2 \ldots$ & 0.20 & 11.086 & 0.384 & $62.24 \pm 0.84$ \\
\hline 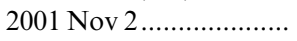 & 0.22 & 11.149 & 0.324 & $52.11 \pm 0.95$ \\
\hline 2001 Nov $6-9$................... & 0.24 & 11.149 & 0.369 & $52.23 \pm 0.50$ \\
\hline 2001 Nov $10-16$............. & 0.26 & 11.171 & 0.355 & $55.88 \pm 0.74$ \\
\hline 2001 Dec $13,14 \ldots \ldots \ldots \ldots$ & 0.35 & 11.149 & 0.243 & $49.31 \pm 1.04$ \\
\hline 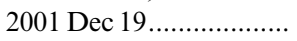 & 0.36 & 11.149 & 0.293 & $49.92 \pm 0.79$ \\
\hline
\end{tabular}

ISI Diameter Measurements of $o$ Cet Made in the Years 1999-2001

a The parameter $A$ is the fraction of the total flux emitted by the stellar disk as defined in eq. (1). It has an uncertainty for all measurements of roughly 0.04 .

The ISI angular diameter measurements made in the years 1999-2001 are displayed in Table 1. The best-fitting diameter and probable error, $2 r \pm 2 \sigma_{r}$, and stellar flux fraction, $A$, are listed in the table. Only data with spatial frequency greater than $1.75 \times 10^{6} \mathrm{rad}^{-1}$ were deemed reliable and included. The probable error of $A$ includes the uncertainty of the calibration and is roughly 0.04 for each measurement. Its value varies substantially with phase and dust formation. Each observation was made in a bandpass centered around the wavelength, $\lambda$, having a full width $\sim 2 \mathrm{~nm}$ (or $0.17 \mathrm{~cm}^{-1}$ ). The standard observation wavelength of $11.149 \mu \mathrm{m}$ was chosen because of the relatively small absorption in Earth's atmosphere or in the circumstellar material at this wavelength. The other observation wavelengths were chosen to probe an observed spectral feature on the star. The increased opacity on these molecular lines causes the star to appear larger at these wavelengths, as evidenced in the table.

\section{INTERPRETATION OF DIAMETER MEASUREMENTS}

The ISI continuum bandpass $(11.149 \mu \mathrm{m})$ diameter of $o$ Cet is higher than most of the shorter wavelength measurements listed in Table 2. Diameters measured in visible and near-IR broad bandpasses can be affected by limb darkening, spectral line contamination, and hot spots in the most general case. The following consideration of the circumstellar environment of Mira will show that the $11 \mu \mathrm{m}$ diameters presented here accurately represent its continuum photosphere to within a few percent.

The dust shell surrounding $o$ Cet, capable of significant scattering and emission of light at some wavelengths, was initially studied with ISI at $11.149 \mu \mathrm{m}$ by Danchi et al. (1994). Dust is believed to form when the temperature in the 
TABLE 2

Various Published Visible and Near-IR $o$ Cet Diameter Measurements

\begin{tabular}{|c|c|c|c|c|c|}
\hline $\begin{array}{c}\lambda \\
(\mu \mathrm{m})\end{array}$ & Phase & $\begin{array}{l}\text { Diameter } \\
\text { (mas) }\end{array}$ & Method $^{\mathrm{a}}$ & Instrument & Reference \\
\hline $0.700 \ldots \ldots$ & $0.05-0.58$ & $41-44$ & $\mathrm{AM}$ & Herschel Telescope & 1 \\
\hline $0.710 \ldots \ldots$. & $0.05-0.58$ & $46-53$ & $\mathrm{AM}$ & Herschel Telescope & 1 \\
\hline $0.800 \ldots \ldots$. & 0.96 & 33 & I & Mark III & 2 \\
\hline $0.800 \ldots \ldots$. & 0.05 & 26 & I & Mark III & 2 \\
\hline $0.800 \ldots \ldots$. & 0.14 & 26 & I & Mark III & 2 \\
\hline $0.833 \ldots \ldots$. & 0.05 & $42.3 \pm 3.4$ & $\mathrm{AM}$ & Herschel Telescope & 1 \\
\hline $0.902 \ldots \ldots .$. & $0.05-0.58$ & $36-38$ & $\mathrm{AM}$ & Herschel Telescope & 1 \\
\hline $0.905 \ldots \ldots$. & 0.67 & $42.0 \pm 1.0^{\mathrm{b}}$ & I & COAST & 3 \\
\hline $1.024 \ldots \ldots$. & 0.67 & $36.3 \pm 1.0^{\mathrm{b}}$ & I & COAST & 3 \\
\hline $1.09 \ldots \ldots \ldots$ & 0.95 & 25 & $\mathrm{AM}$ & Keck Telescope & 4 \\
\hline $1.290 \ldots \ldots$. & 0.67 & $31.3 \pm 0.5^{b}$ & I & COAST & 3 \\
\hline $1.28 \ldots \ldots \ldots$ & 0.95 & 20 & $\mathrm{AM}$ & Keck Telescope & 4 \\
\hline $1.64 \ldots \ldots \ldots$ & 0.95 & 28.5 & $\mathrm{AM}$ & Keck Telescope & 4 \\
\hline $2.12 \ldots \ldots \ldots$ & 0.95 & 34 & $\mathrm{AM}$ & Keck Telescope & 4 \\
\hline $2.2 \ldots \ldots \ldots \ldots$ & 0.94 & $28.8 \pm 0.1$ & I & IOTA & 5 \\
\hline $2.2 \ldots \ldots \ldots \ldots$ & $0.23-0.36$ & $36.1 \pm 1.4$ & I & Michelson Array & 6 \\
\hline $3.09 \ldots \ldots \ldots$ & 0.95 & 60 & $\mathrm{AM}$ & Keck Telescope & 4 \\
\hline $3.75 \ldots \ldots \ldots$ & 0.98 & $43.5 \pm 0.2$ & I & IOTA & 5 \\
\hline Several .... & $0.5-1.1$ & 24-39 & $\mathrm{P}$ & Several & 7 \\
\hline
\end{tabular}

a AM: aperture masking; I: interferometry; P: infrared photometry.

b Implied uniform disk diameter. The reference listed a Gaussian FWHM, which was a factor of $\sim 1.61$ times smaller.

ReFerences.- (1) Haniff, Sholz, \& Tuthill 1995; (2) Quirrenbach et al. 1992; (3) Young et al. 1999; (4) Tuthill et al. 1999a; (5) Mennesson et al. 2000; (6) Ridgway et al. 1992; (7) Mahler, Wasatonic, \& Guinan 1997.

circumstellar environment drops below the temperature of condensation $^{3}$ and takes on an approximately $r^{-2}$ distribution as it is blown outward by the stellar wind. The inner radius of the dust shell was measured by Danchi et al. (1994) to be $\sim 2 R_{*}$ (where $R_{*}$ is the stellar radius), in reasonable agreement with dynamic ${ }^{4}$ Mira model atmospheres (Höfner et al. 1998). The presence of a dust shell may introduce some bias to the measured diameter. However, a symmetric dust shell having an $r^{-2}$ distribution and contributing $65 \%$ of the total flux will have an effect on the best-fit diameter of less than $\pm 3 \%$, provided the inner radius of the dust shell is greater than $1.5 R_{*}$. For the specific case of a dust shell with an inner radius of $2 R_{*}$, the best-fitting diameter will be $3 \%$ larger than the actual diameter. The calculation of these values is discussed in Weiner (2002).

The actual dust shell of $o$ Cet may be more complicated than this simple model. In the very dynamic and unstable atmospheres surrounding long-period variables, sizable asymmetries may develop as the dust forms (Woitke, Sedlmayr, \& Lopez 2000). Evidence for "clumps" in the dust shell of $o$ Cet is presented by Lopez et al. (1997). Models containing large clumps of dust with densities $\sim 100$ times the surrounding dust density and contributing up to $5 \%$ of the stellar flux were successfully fitted to $11 \mu \mathrm{m}$ interferometric observations. A small clump of dust (Gaussian in shape with a FWHM of $0.6 R_{*}$ ) located at $2 R_{*}$ to the side of the star and contributing $15 \%$ of the stellar flux will reduce

\footnotetext{
3 Typically, around $1000 \mathrm{~K}$, although somewhat density dependent (Suh 1999).

${ }^{4}$ Dynamic models predict postshock cooling to be strong enough to allow dust formation to occur at radii having equilibrium temperatures above the dust condensation temperature.
}

the apparent diameter by $2.2 \%-4.1 \%$, depending on the position angle. Similarly, if the dust clump is located at $1 R_{*}$ to the side, or equivalently, in front of the star and offset from the line of sight by $1 R_{*}$, the apparent diameter will be reduced by up to $2.6 \%$ or enlarged by up to $4.1 \%$, depending on position angle. Because of the lack of evidence at $11 \mu \mathrm{m}$ for dust clumps contributing more than $5 \%$ of the stellar flux, we take the above estimates to be an upper limit to the effect dust asymmetries and/or clumping may have on diameter measurements.

The low temperatures and high densities that exist in the atmospheres of Miras induce a variety of di- and polyatomic molecules to form. These molecules, most prominently $\mathrm{CO}$, $\mathrm{TiO}, \mathrm{SiO}$, and $\mathrm{H}_{2} \mathrm{O}$, often have "forests" of spectral lines, which contribute significant opacity to the circumstellar region in some wavelength regions. There is ample evidence that the spectra of AGB stars are greatly affected by TiO and $\mathrm{H}_{2} \mathrm{O}$ line opacity (see Tsuji 2000, Wing 1997, and references therein). There is also significant evidence that the measured sizes of stars are affected by the presence of molecules. In general, molecular transition opacity can either decrease the apparent size of a star through the effect of limb darkening or increase it if the material is opaque or located at large radii. Diameter measurements were made for a number of stars with the Mark III interferometer at both 712 and $754 \mathrm{~nm}$ (Quirrenbach et al. 2001). It was found that the $712 \mathrm{~nm}$ sizes were systematically larger because of the strong TiO bands present at this wavelength and that the effect was most pronounced for stars with low effective temperatures. The effect of spectral lines is also evident in the stellar diameters of Tuthill, Haniff, \& Baldwin (1999a). Between 1.3 and $3.1 \mu \mathrm{m}$, the apparent size of $o$ Cet was seen to increase from 21 to 60 mas because of the presence of 

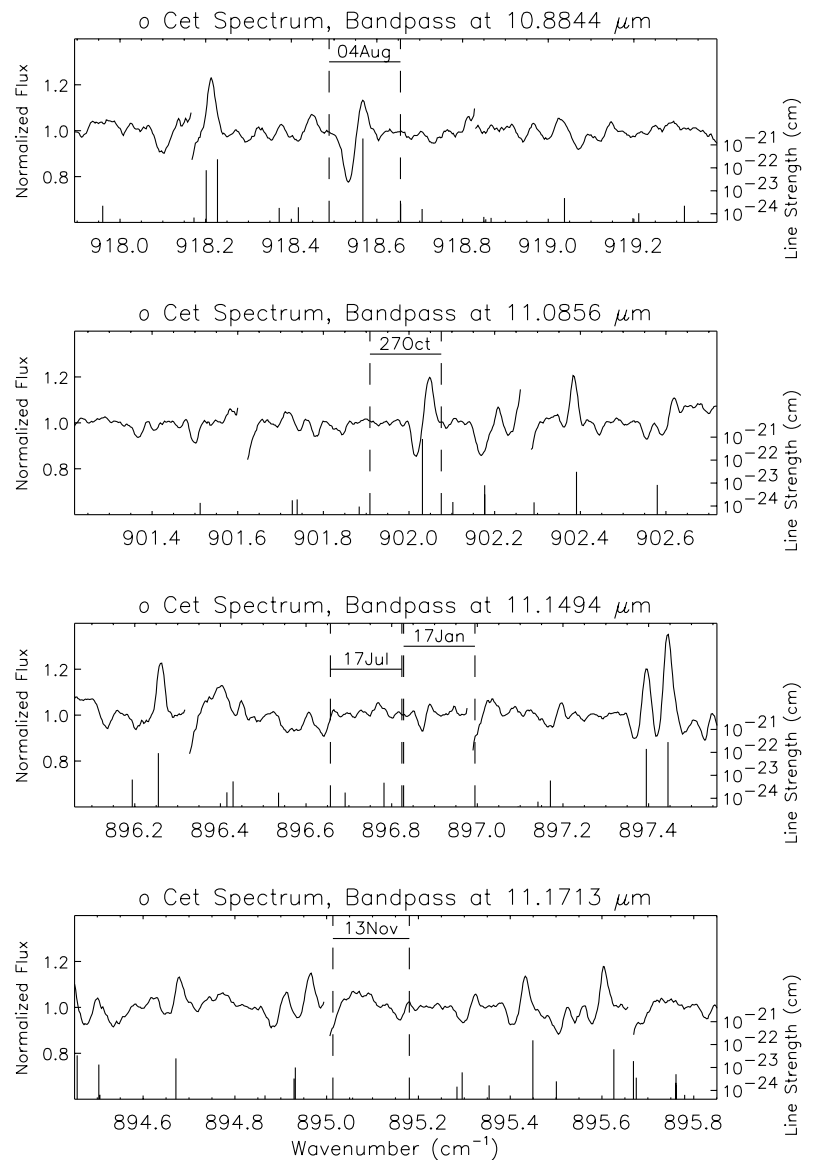

FIG. 2.- Spectra of $o$ Cet near ISI bandpasses. Dashed vertical lines mark the approximate edges of the bandpass at the date shown. Vertical bars mark prominent $\mathrm{H}_{2} \mathrm{O}$ transitions (with line strength given by right axis, assuming gas temperature of $1000 \mathrm{~K}$ and redshift of $83 \mathrm{~km} \mathrm{~s}^{-1}$ ).

strong $\mathrm{TiO}$ and $\mathrm{H}_{2} \mathrm{O}$ bands. The importance of unbiased continuum diameters makes avoiding spectral lines in stellar size measurements quite important.

The ISI records visibilities in various narrow $(\sim 0.17$ $\mathrm{cm}^{-1}$ ) bandpasses. Comparison of available wavelength regions with high-resolution spectra allows for the selection of line-free observing regions in which to make continuum diameter measurements. Conversely, the wide bandpasses commonly used by other instruments to measure stellar diameters tend to average in some unavoidable spectral lines. High-resolution spectra of $o$ Cet at phase 0.36, recorded at the IRTF telescope using the TEXES instrument ${ }^{5}$ are displayed in Figure 2. The flux is plotted as a function of wavelength, with dashed vertical lines marking the approximate edges of the ISI bandpasses as they would appear on the date shown. ${ }^{6}$ Vertical bars marking $\mathrm{H}_{2} \mathrm{O}$ transitions with line strength ${ }^{7}$ given by the right axis are evident in the spectra. The lack of strong lines within the bandpass at $11.149 \mu \mathrm{m}$ implies that the effect of any spectral lines on

\footnotetext{
${ }^{5}$ The observations were carried out by John Lacy and Matt Richter, and a description of the instrument can be found in Lacy et al. (2002).

${ }^{6}$ For the bandpass at $11.149 \mu \mathrm{m}$, the two dates listed are extrema of the Earth's motion toward and away from $o$ Cet. The bandpass on any other observing date lies between these two.

${ }^{7}$ Line strengths were calculated from the HITRAN/HITEMP database, Rothman et al. (1992) assuming a temperature of $1000 \mathrm{~K}$ and a redshift of $83 \mathrm{~km} \mathrm{~s}^{-1}$
}

the size measurements in this bandpass are small. Assuming reasonable gas parameters, the presence of any absorption lines shallower than $5 \%$ that may not be evident in the spectra should affect the measured diameter by less than $1 \%$ (discussion in Weiner 2002).

In addition to that of dust particles and spectral lines, the continuum opacity of the gas making up the photosphere also has some wavelength dependence. Hence, some variation in apparent diameter with wavelength is expected, even in the absence of spectral lines. The magnitude of this difference is determined by the "steepness" of the density falloff at the surface of the star and can be estimated from a model of the stellar structure.

The density stratification of a Mira variable departs significantly from hydrostatic equilibrium as a result of the periodic passage of shock waves. The temperature and density for $o$ Cet, taken from the spherically symmetric dynamic model D27520 of Bessell, Scholz, \& Wood (1996), ${ }^{8}$ and corresponding to visual phase 0.0 , were used to calculate the continuum opacity and the resulting intensity distribution of the Mira model. The total opacity from continuum sources, including contributions from $\mathrm{H}_{\mathrm{ff}}^{-}, \mathrm{H}_{2 \mathrm{ff}}^{-}, \mathrm{He}_{\mathrm{ff}}^{-}, \mathrm{H}_{\mathrm{ff}}, \mathrm{K}_{\mathrm{ff}}$, $\mathrm{Na}_{\mathrm{ff}}, \mathrm{Ca}_{\mathrm{ff}}$, and $\mathrm{Al}_{\mathrm{ff}}$ processes ${ }^{9}$ and from Thomson scattering, was calculated for the range of temperatures and densities in the model atmosphere, assuming solar abundances. The ionization states and free electron density used were obtained through a simultaneous solution of the ionization equation for $\mathrm{H}, \mathrm{He}, \mathrm{K}, \mathrm{Na}, \mathrm{Ca}$, and $\mathrm{Al}$ assuming local thermodynamic equilibrium. ${ }^{10}$ The intensity profiles were calculated by integrating a blackbody source function over the calculated opacity of the model.

The density and temperature structure taken from Bessell et al. (1996) and the calculated $11.149 \mu \mathrm{m}$ opacity, $-d \tau / d r$, are plotted in Figure 3 as a function of Rosseland opacity. The integrated $11.149 \mu \mathrm{m}$ optical depth (assuming only the opacity sources listed above), as well as the monochromatic optical depths at $1.04 \mu \mathrm{m}, 712 \mathrm{~nm}$, and $653 \mathrm{~nm}$ (given by Bessell et al. 1996), are indicated by the horizontal scales. The monochromatic radius of the Mira model at $11.149 \mu \mathrm{m}$, defined as the radius at which $\tau_{11.149} \mu \mathrm{m}=1$, is only $0.06 \%$ larger than the Rosseland radius (the radius at which $\tau_{\text {Rosseland }}$ is unity). Monochromatic radii and uniform disk apparent radii at a variety of wavelengths for the D27520 model are given in Hofmann, Scholz, \& Wood (1998). The $11.149 \mu \mathrm{m}$ monochromatic radius calculated here is only $2 \%$ larger than the monochromatic radius given for continuum bands at $1.63 \mu \mathrm{m}$ and $2.2 \mu \mathrm{m}$ and smaller than all of the monochromatic radii at wavelengths shorter than $1 \mu \mathrm{m}$.

The $11.149 \mu \mathrm{m}$ intensity distribution resulting from this model is shown in Figure 4. The apparent size of the star, resulting from a uniform disk fit of the calculated visibilities, is $255 R_{\odot}$, which is $3.9 \%$ greater than the Rosseland diameter according to the model of Bessell et al. (1996). Compared to predicted uniform disk sizes at other wavelengths (given by Hofmann et al. 1998), this value is similar to some of the

\footnotetext{
8 The density and temperature as a function of radius was estimated from Fig. 4 of Bessell et al. (1996).

${ }^{9}$ Theoretical cross sections from Dalgarno \& Lane (1966) were used. $\mathrm{H}_{\mathrm{ff}}^{-}$is the dominant opacity for densities greater than $\sim 10^{12} \mathrm{~cm}^{-3}$ and temperatures between $\sim 2000 \mathrm{~K}$ and $\sim 5000 \mathrm{~K}$, which characterize the stellar surface.

${ }^{10}$ Nonequilibrium conditions likely exist at the shock front in Miras but do not contribute substantial $11 \mu \mathrm{m}$ optical depth. A discussion based on the shock models of Fox \& Wood (1985) can be found in Weiner (2002).
} 


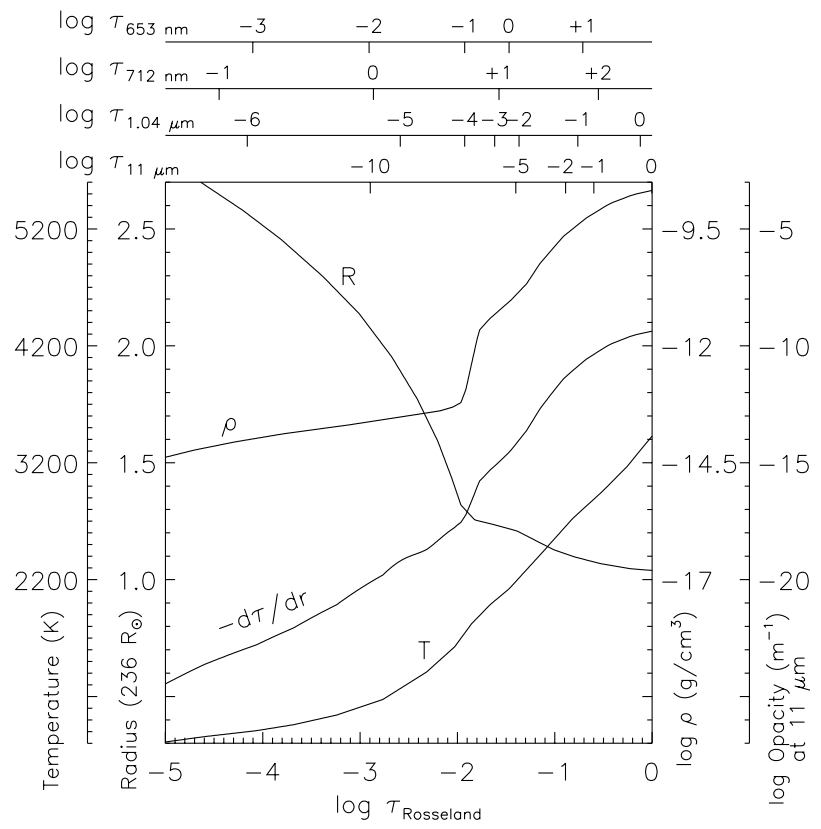

Fig. 3.-Density and temperature Mira structure from D27520 model of Bessell et al. (1996) at visual phase 0.0 and calculated $11.149 \mu \mathrm{m}$ LTE opacity (from continuum sources) as a function of Rosseland optical depth. The integrated $11.149 \mu \mathrm{m}$ optical depth, as well as the $1.04 \mu \mathrm{m}, 712 \mathrm{~nm}$, and 653 $\mathrm{nm}$ monochromatic optical depths from Bessell et al. (1996) are indicated on the horizontal scales.

visible sizes, $11 \%$ larger than the $2.2 \mu \mathrm{m}$ apparent size, and $12 \%$ larger than the $1.63 \mu \mathrm{m}$ apparent size. It is interesting to note that the $11 \mu \mathrm{m}$ uniform disk radius is larger than the $11 \mu \mathrm{m}$ monochromatic radius (by $3.9 \%$ ) because of the contribution of small opacity sources near the "wing" of the star and the relatively low dependence of intensity on temperature at this wavelength. In the near-infrared, however, the situation is reversed, since intensity is much more sensitive to temperature, causing the intensity distribution to be strongly limb darkened. This causes near-IR uniform disk sizes to be smaller than their corresponding monochromatic radii. As a result, monochromatic radii at $11 \mu \mathrm{m}$ may be

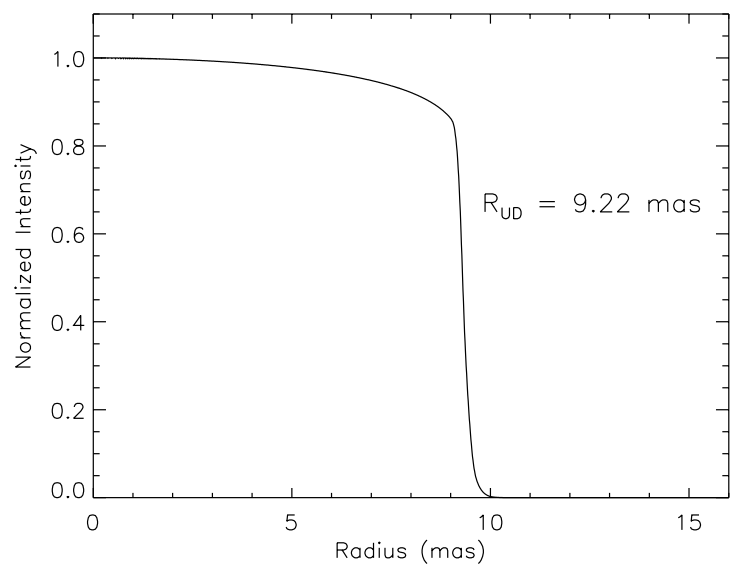

FIG. 4.-Normalized $11.149 \mu \mathrm{m}$ intensity profile calculated from the Mira model of Bessell et al. (1996) at maximum phase including only continuum opacity sources in LTE. The apparent radius resulting from a uniform disk fitted to the visibilities calculated from the profile is 9.22 mas. A distance of $128 \mathrm{pc}$ was assumed for conversion to an angular radial scale. only $2 \%$ larger than those for the near-IR, while the apparent uniform disk size is $12 \%$ larger. At any rate, according to dynamic models of Mira stellar structure, the ISI diameters are predicted to be $\sim 4 \%$ larger than the Rosseland diameter and on the order of $10 \%$ larger than apparent sizes measured in near-infrared near-continuum bands. In fact, ISI-measured diameters are much larger than this.

Despite a systematic consideration of the stellar atmosphere, no effect capable of enlarging $11 \mu \mathrm{m}$ apparent sizes by the observed factor of $\sim 2$ was discovered. Dust, molecular transitions, and differential continuum opacities can, from present theoretical models, be ruled out as causes. It can be concluded either that the small sizes seen with broad bandpasses in the near-IR have been incorrectly identified with continuum diameters (when they actually refer to smaller-scale features on the stellar surface) or that current dynamic Mira models predict too steep a density gradient in the stellar photosphere.

The debate surrounding the pulsation mode of Miras is tied to the stellar size as well. An observationally derived period-radius relationship can be used to differentiate between fundamental and overtone mode pulsation in Miras. However, there is some disagreement among pulsation theories concerning stellar size. The maximum diameter of a fundamental-mode 332 day period Mira from Bowen (1990) is 20 mas (assuming a distance of $128 \mathrm{pc}$ ), whereas Tuchman (1999) predicts a value of 34 mas. Thus, for some models, the lowest of the observed $o$ Cet diameters (made at near-IR wavelengths) are consistent with fundamentalmode pulsation. However, present theory and Mira's $11 \mu \mathrm{m}$ diameter imply an overtone pulsation.

\section{VARIATION OF THE DIAMETER OF $o$ CET}

Variations in the diameter of $o$ Cet were observed in the continuum bandpass at $11.149 \mu \mathrm{m}$ over the three years 1999-2001. Statistically significant changes in the size of $o$ Cet with phase and from cycle to cycle were seen. The diameter ${ }^{11}$ of $o$ Cet as a function of date is plotted in Figure 5. The clearest observed variation with phase resulted from the numerous 2001 measurements. The 1999 data agree well with the 2000 diameters. However, the 2001 measurements were taken using a different baseline than the previous two years, and the measured diameter is larger by $\sim 5.4$ mas (or $11.1 \%$ ). The $1999 / 2000$ baseline was $56 \mathrm{~m}$ and oriented $249^{\circ}$ east of north, while the 2001 baseline was $51 \mathrm{~m}$ and $285^{\circ}$ east of north, corresponding to a change in position angle of 36.5. The curve in Figure 5 that fits the observations is the best-fitting sinusoid, having an amplitude of 6 mas around an average value of 42.6 mas for the 1999/2000 years and 48 mas for 2001. The maximum size is obtained at phase 0.135 . By comparison, the size of $\alpha$ Ori was not seen to change significantly over the same time period using the same baselines. The diameter of $\alpha$ Ori as a function of date is plotted in Figure 6. Deviations in the diameter from its average value are no more than $2 \sigma$ for any of the 10 measurements. Some variability in the size of $\alpha$ Ori might be thought to be present but is less than about $\pm 2 \%$ in any case. The stability

\footnotetext{
${ }^{11}$ For consistency between dates, all visibility data below a spatial frequency of $2.5 \times 10^{6} \mathrm{rad}^{-1}$ was ignored since variations in the dust shell may affect low spatial frequency visibilities somewhat. Because of this, some slight differences with respect to Table 1 are introduced.
} 

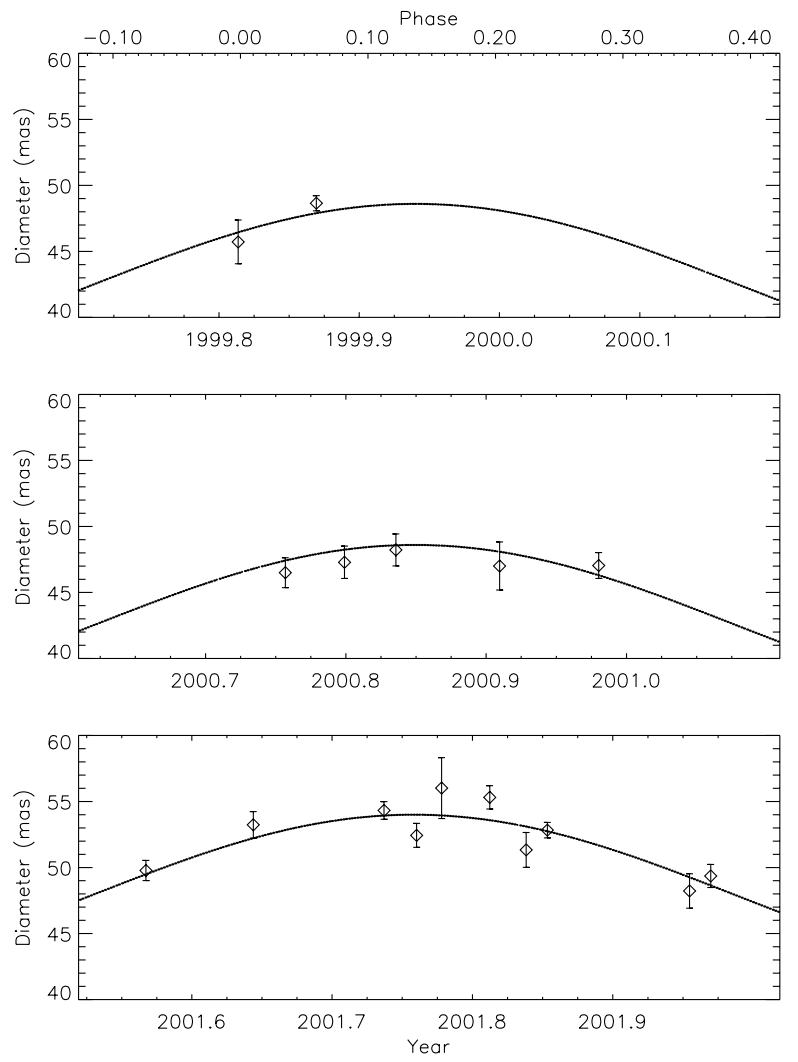

Fig. 5.-Variation of the diameter of $o$ Cet with phase (given by the top axis) and date. Also plotted is the best-fitting sinusoid having a peakto-peak amplitude of 12 mas around an average diameter of 48 mas for 2001 and 42.6 mas for 1999 and 2000 and obtaining maximum size at phase 0.135 .

of the diameter of $\alpha$ Ori implies that the variations exhibited by $o$ Cet, including the variation with baseline orientation, are not instrumental in nature.

The $11 \%$ increase in the size of Mira between 2000 and 2001 is possibly a nonperiodic timelike change in size such as those observed in $o$ Cet in the visible by Tuthill, Haniff, \& Baldwin (1995). However, in that paper, the size variations

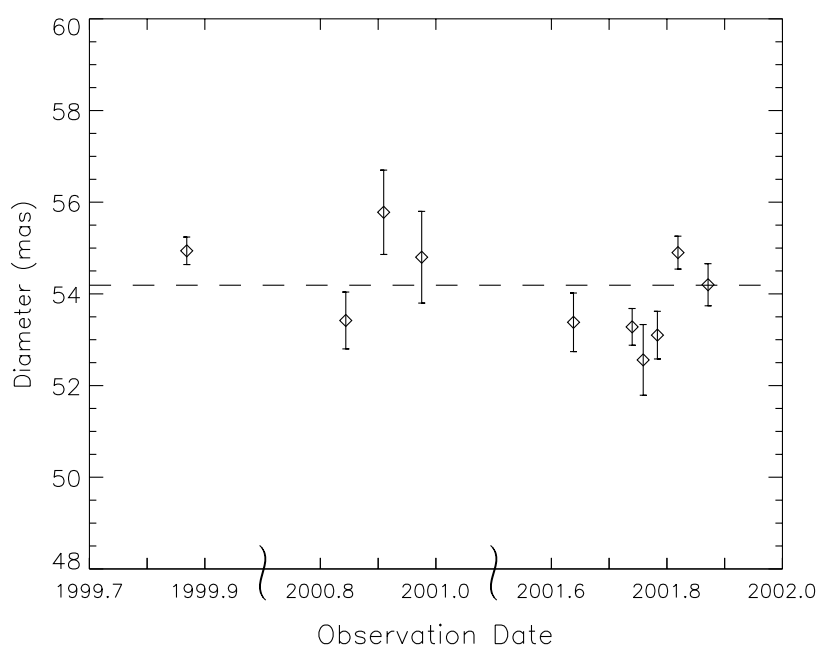

FIG. 6.-Variation of the diameter of $\alpha$ Ori with date. Fluctuations in the diameter measurements of about $2 \%$ from average are seen. were most pronounced in the strongest $\mathrm{TiO}$ absorption bands and were identified with exophotospheric layers. ISI diameter measurements are believed to represent the stellar continuum photosphere. The size increase is more likely attributed to a spacelike asymmetry in the star that was manifested as a size change because of the different position angles of the 2000 and 2001 baselines. Elongation with an axial ratio as low as 0.77 in $o$ Cet in the visible has been reported by Karovska (1997). A uniform-intensity elliptical stellar disk having axial ratio less than 0.83 could be responsible for the observed increase in diameter between the $1999 / 2000$ and 2001 baselines. Elongation in Mira variables could be due to excitation of a nonradial mode of pulsation or possibly to rotation. Hot spots on the surface of $o$ Cet also would be evidenced (to first order) as elongation, provided they are not centered.

The observed change in diameter of $o$ Cet with phase fits theory and expectations rather well. Between phases -0.08 and 0.15 , the size was seen to increase by about $11 \%$, then decrease by $13 \%$ by phase 0.36 . The best-fitting sinusoidal variation was seen to have an amplitude of 6 mas, corresponding to a change of $\pm 12.5 \%$ in a cycle.

Predictions have been made concerning the magnitude of the radial pulsation in Miras. The standard Mira model from Bowen (1990) $\left(1 M_{\odot}, 320\right.$ day period, $\left.T_{\text {eff }}=3000 \mathrm{~K}\right)$ undergoes a pulsation of the Lagrangian zone corresponding to its photospheric shell of $\pm 11.7 \%$, in close agreement with ISI data. Bessell et al. (1996) predict changes of about $\pm 15 \%$ in the Rosseland radii of a fundamental-mode Mira. In addition, the maximum radius is predicted to be near visible phase 0.2 , in close agreement with ISI data. The nonlinear Mira models of Ya'ari \& Tuchman (1999) predict a change in surface radii by about a factor of 2 through an entire cycle, although most of the variation occurs near minimum radii. In the range of phases covered by ISI measurements, the predicted radius changes by only $\sim 13 \%$, also consistent with the observations.

In addition to the predictions, there have been a few variations in the apparent size of Miras observed at shorter wavelengths. The Mira R Leo appeared to change in size by $\sim 30 \%$ at 830 and $940 \mathrm{~nm}$ (Burns et al. 1998), although maximum size was obtained at phase 0.5 , indicating a large phase lag between the photospheric pulsation and the observed size. Burns et al. (1998) conclude that the observations reflect the variation of the $\mathrm{TiO}$ bands due to pulsation, rather than the photospheric continuum. Young et al. (1999) report variation of the apparent diameter of $\chi$ Cyg by $\sim 45 \%$ at $905 \mathrm{~nm}$, but little change in size at $1290 \mathrm{~nm}$. Varying molecular bands are believed to cause the size change at $905 \mathrm{~nm}$. The $K$ band uniform disk size of R Leo was seen to increase by $8.8 \%$ between phases 0.24 and 0.28 (Perrin et al. 1999), although the measurements were almost a year apart in successive cycles. Thompson, CreechEakman, \& van Belle (2002) report cyclic variations in the diameter of RZ Peg, obtaining its maximum at phase 0.28 in the $K$ band. Some variations in the size of Miras are evident in Van Belle et al. (1996) as well.

The ISI $11 \mu \mathrm{m}$ diameters are believed to be a conclusive demonstration of stellar pulsation in the continuum photosphere of a Mira. The measurements largely avoid effects of spectral lines and dust, which strongly affect broadband measurements at shorter wavelengths. The magnitude of the change observed and the phase at which it occurs are consistent with predictions of the dynamic Mira models. 
TABLE 3

Diameter Measurements of $o$ Cet on Spectral Lines

\begin{tabular}{|c|c|c|c|c|c|c|}
\hline $\begin{array}{c}\lambda \\
(\mu \mathrm{m})\end{array}$ & Dates & Phase & $\begin{array}{l}\text { Observed Size Change } \\
\qquad D_{\lambda} / D_{11.149 \mu \mathrm{m}} \\
(\%)\end{array}$ & $\begin{array}{c}\text { Derived } \mathrm{H}_{2} \mathrm{O} \\
\text { Region } \mathrm{Radius}^{\mathrm{a}} \\
\qquad\left(R_{*}\right)\end{array}$ & $\begin{array}{l}\text { Line Strength } \\
\text { at } 1300 \mathrm{~K} \\
(\mathrm{~cm})\end{array}$ & $\begin{array}{l}\text { Implied Line-of-Sight } \\
\text { Column Density } \\
\left(\mathrm{cm}^{-2}\right)\end{array}$ \\
\hline $10.884 \ldots$. & 2001 Aug 1-8 & 0.95 & $+12.8 \pm 2.9$ & $1.70 \pm 0.14$ & $4.56 \times 10^{-21}$ & $4.0 \times 10^{18}$ \\
\hline $11.086 \ldots$ & 2001 Oct 25,26, Nov 2 & 0.20 & $+15.0 \pm 1.8$ & $1.82 \pm 0.25$ & $1.96 \times 10^{-21}$ & $9.3 \times 10^{18}$ \\
\hline $11.171 \ldots$. & 2001 Nov $10-16$ & 0.26 & $+7.0 \pm 1.6$ & $1.41 \pm 0.07^{\mathrm{b}}$ & $8.90 \times 10^{-23}$ & $2.0 \times 10^{20 b}$ \\
\hline
\end{tabular}

a A temperature of $1300 \pm 200 \mathrm{~K}$ was assumed for the $\mathrm{H}_{2} \mathrm{O}$ gas.

${ }^{b}$ May not be accurate. This spectral line was only partially within the bandpass.

\section{THE DIAMETER OF $o$ CET ON $\mathrm{H}_{2} \mathrm{O}$ SPECTRAL LINES}

Strong $\mathrm{H}_{2} \mathrm{O}$ spectral lines were identified in the spectra of $o$ Cet at phase 0.36 as displayed in Figure 2. A characteristic absorption line with depth up to $\sim 20 \%$ was observed at a wavenumber $0.033 \mathrm{~cm}^{-1}$ (corresponding to a redshift of 11 $\mathrm{km} \mathrm{s}^{-1}$ ) lower than each emission peak that has height up to $\sim 40 \%$. The excitation temperature ${ }^{12}$ of the $\mathrm{H}_{2} \mathrm{O}$ in the absorption lines is $1700 \mathrm{~K}$ with a column density roughly $2 \times 10^{18} \mathrm{~cm}^{-2}$. The emission lines have an excitation temperature of $1075 \mathrm{~K}$ and a column density of about $10^{19}$ $\mathrm{cm}^{-2}$. These spectral features observed in $o$ Cet could be produced by $\mathrm{H}_{2} \mathrm{O}$ both in a region in front of the star having a temperature cooler than the photosphere (producing the absorption) and in an even cooler region at larger radii, producing emission and expanding relative to the inner gas.

As mentioned before, the $11.149 \mu \mathrm{m}$ band does not appear to contain any spectral lines stronger than about 5\% and the strongest $\mathrm{H}_{2} \mathrm{O}$ transition within the bandpass has strength $\sim 5 \times 10^{-24} \mathrm{~cm}$ (as compared to $10^{-22}$ to $10^{-21} \mathrm{~cm}$ for the observed strong lines). An upper limit of $\sim 1 \%$ is put on any error in the measured $11.149 \mu \mathrm{m}$ diameter because of spectral contamination (further discussion in Weiner 2002). Each of the three other bandpasses contain strong $\mathrm{H}_{2} \mathrm{O}$ transitions, which are evident in both the spectra and the diameter measurements in these bands. ${ }^{13}$ The measured size changes (the ratio of the diameter measured in each of these bandpasses to the continuum $11.149 \mu \mathrm{m}$ diameters at nearby dates) are listed in Table 3.

The size of the emitting molecular region can be calculated from the diameter measured on the spectral line if it is assumed that the $\mathrm{H}_{2} \mathrm{O}$ is optically thick out to some radius on the emission line and that the apparent size is effected only negligibly in the absorption line. Using this assumption, the intensity profile averaged over the bandpass is the sum of a stellar-sized uniform disk in the continuum portion of the bandpass and a gas region-sized uniform disk on the emission line. The visibilities resulting from such a profile are best fitted by a uniform disk of radius somewhat larger than the continuum value. In this way, the relative size increase in a bandpass containing an $\mathrm{H}_{2} \mathrm{O}$ line can be used to deduce the radius of the $\mathrm{H}_{2} \mathrm{O}$ gas region. For this, the stellar temperature was taken to be $2500 \mathrm{~K}$, the $\mathrm{H}_{2} \mathrm{O}$ gas was

\footnotetext{
12 The excitation temperature was estimated by comparing the depth of observed lines with line strengths calculated from HITRAN/HITEMP (Rothman et al. 1992) for a range of temperatures.

${ }^{13}$ The spectral line in the bandpass at $11.171 \mu \mathrm{m}$ shown in Figure 2 appears outside of the bandpass but may be partially contained within it because the bandpass has edges that are more gradual than those shown.
}

assumed to be at $1300 \pm 200 \mathrm{~K},{ }^{14}$ and the width of the emission line was estimated at one-fourth of the ISI bandwidth. The derived radius of the gas region for each off-continuum diameter measurement is listed in Table 3.

Some information regarding the amount of $\mathrm{H}_{2} \mathrm{O}$ gas in the stellar atmosphere can also be estimated from the above analysis. The gas distribution will, of course, not have a sharp edge as modeled, but rather the intensity profile will fall off at the radius where the line-of-sight optical depth becomes less than unity. So, it can be assumed that an optical path aimed at the edge of the disklike gas region above has optical depth unity. Since the $\mathrm{H}_{2} \mathrm{O}$ transition strength is known, ${ }^{15}$ the total molecular column density along the line of sight aimed at the edge of the gas region can be calculated. These column densities are listed in Table 3. Since these path lengths (through the atmosphere) are on the order of $10^{14} \mathrm{~cm}$, we conclude that $\mathrm{H}_{2} \mathrm{O}$ densities on the order of $10^{5} \mathrm{~cm}^{-3}$ exist near 1.75 stellar radii.

\section{CONCLUSIONS}

Heterodyne interferometry has been employed at $11 \mu \mathrm{m}$ to measure the size of $o$ Cet with a precision of about $1 \%$. The visibilities are consistent with a uniform disk intensity distribution having a diameter greater than most previous diameter measurements in the visible and near-infrared. It can be concluded that dust surrounding $o$ Cet, which produces significant $11 \mu \mathrm{m}$ flux, does not bias the diameter measurements at this wavelength by more than $3 \%$ in either direction. High-resolution spectra of $o$ Cet were taken near $11 \mu \mathrm{m}$, and it was found that the bandpass at $11.149 \mu \mathrm{m}$ does not contain any strong spectral lines. An upper limit of about $1 \%$ can be placed on the effect spectral-line opacity may have on diameter measurements in the continuum $11.149 \mu \mathrm{m}$ band. The conclusion is drawn that the 11.149 $\mu \mathrm{m}$ measurements characterize the continuum photosphere of $o$ Cet.

Dynamic Mira models, including the models of Bessell et al. (1996) considered here, predict extremely extended density and temperature structure in the stellar atmosphere, resulting in a continuum photosphere having a radius that varies with wavelength. However, the difference between $11 \mu \mathrm{m}$ apparent sizes (calculated from continuum opacity sources only) and Rosseland diameters is estimated to be

\footnotetext{
${ }^{14}$ The gas was seen to have an excitation temperature of $1075 \mathrm{~K}$ at phase 0.36 , but these diameter measurements were taken closer to maximum. The large uncertainty in this temperature is responsible for the large uncertainties in the derived gas region radius listed in Table 3.

${ }^{15}$ The HITRAN/HITEMP database was used to calculate line strength at $1300 \mathrm{~K}$.
} 
only $\sim 4 \%$. In fact, the observed ISI $11 \mu \mathrm{m}$ size is almost twice the near-infrared apparent sizes. It is concluded that either the small sizes observed in the near-infrared have been incorrectly assumed to represent photospheric stellar radii or that the dynamic models considered predict insufficient atmospheric extension in Miras.

Stellar pulsation was observed to occur in $o$ Cet, resulting in a variation in the continuum size of $+11 \%$ between phase -0.08 and 0.15 and $-13 \%$ between phase 0.15 and 0.36 . Also, an $11 \%$ increase in size was observed between the 1999/2000 sizes and the 2001 sizes. This was most likely caused by an asymmetry or elongation in $o$ Cet that was manifested as a size change when the position angle of the baseline was changed by $36^{\circ} .5$ in 2001 June.
The location of molecular $\mathrm{H}_{2} \mathrm{O}$ gas in the stellar atmosphere was probed by performing diameter measurements in a bandpass known to contain strong $\mathrm{H}_{2} \mathrm{O}$ spectral features. Consistently larger sizes were observed in such bandpasses. Estimates imply $\mathrm{H}_{2} \mathrm{O}$ densities on the order of $10^{5}$ $\mathrm{cm}^{-3}$ near 1.75 stellar radii in order to explain the up to $15 \%$ size increases observed on $\mathrm{H}_{2} \mathrm{O}$ lines.

This work was supported in part by the National Science Foundation (AST 97-31625) and the Office of Naval Research (N00014-89-J-1583). We are grateful to Matt Richter and John Lacy for their privately supplied mid-IR spectrum of $o$ Cet.
Bessell, M. S., Scholz, M., \& Wood, P. R. 1996, A\&A, 307, 481

Bevington, P. R. 1969, Data Reduction and Error Analysis for the Physical Sciences (Boston: McGraw-Hill)

Bowen, G. H. 1990, in Numerical Modeling of Nonlinear Stellar Pulsations Problems and Prospects, ed. J. R. Buchler (Dordrecht: Kluwer), 155

Burns, D., et al. 1998, MNRAS, 297, 462

Dalgarno, A., \& Lane, N. F. 1966, ApJ, 145, 623

Danchi, W. C., Bester, M., Degiacomi, C. G., Greenhill, L. J., \& Townes, C. H. 1994, AJ, 107, 1469

Fox, M. W., \& Wood, P. R. 1985, ApJ, 297, 455

Hale, D. D. S., et al. 2000, ApJ, 537, 998

Haniff, C. A., Scholz, M., \& Tuthill, P. G. 1995, MNRAS, 276, 640

Hofmann, K.-H., Scholz, M., \& Wood, P. R. 1998, A\&A, 339, 846

Höfner, S., J J rgensen, U. G., Loidl, R., \& Aringer, B. 1998, A\&A, 340, 497

Josselin, E., Mauron, N., Planesas, P., \& Bachiller, R. 2000, A\&A, 362, 255

Karovska, M. 1997, J. AAVSO, 25, 75

Lacy, J. H., Richter, M. J., Greathouse, T. K., Jaffe, D. T., \& Zhu, Q. 2002, PASP, 114, 153

Lopez, B., et al. 1997, ApJ, 488, 807

Mahler, T. A., Wasatonic, R., \& Guinan, E. F. 1997, Inf. Bull. Variable Stars, 4500,1

Mennesson, B., et al. 2000, Proc. SPIE, 4006, 481

Perrin, G., et al. 1999, A\&A, 345, 221

Quirrenbach, A., Mozurkewich, D., Armstrong, J. T., Johnston, K. J., Colavita, M. M., \& Shao, M. 1992, A\&A, 259, L19

Quirrenbach, A., et al. 2001, in Proc. IAU Symp. 205, Galaxies and Their Constituents at the Highest Angular Resolutions, ed. R. T. Schilizzi,

S. N. Vogel, F. Paresce, \& M. Elvis (San Francisco: ASP), 304

Reid, M. J., \& Menten, K. M. 1997, ApJ, 476, 327

\section{EFERENCES}

Ridgway, S. T., Benson, J. A., Dyck, H. M., Townsley, L. K., \& Hermann, R. A. 1992, ÄJ, 104, 2224

Rothman, L. S., et al. 1992, J. Quant. Spectrosc. Radiat. Transfer, 48, 469

Schwarzschild, M. 1975, ApJ, 195, 137

Suh, K.-W. 1999, MNRAS, 304, 389

Thompson, R. R., Creech-Eakman, M. J., \& van Belle, G. T. 2002, ApJ, 577,447

Tsuji, T. 2000, ApJ, 538, 801

Tuchman, Y. 1999, in IAU Symp. 191, Asymptotic Giant Branch Stars, ed. T. Le Bertre, A. Lebre, \& C. Waelkens (San Francisco: ASP), 123

Tuthill, P. G., Haniff, C. A., \& Baldwin, J. E. 1995, MNRAS, 277, 1541 1999a, MNRAS, 306, 353

Tuthill, P. G., Monnier, J. D., \& Danchi, W. C. 1999b, in ASP Conf. Ser 194, Working on the Fringe: Optical and IR Interferometry from Ground and Space, ed. S. Unwin \& R. Stachnik (San Francisco: ASP), 188

van Belle, G. T., Dyck, H. M., Benson, J. A., \& Lacasse, M. G. 1996, AJ, 112,2147

Weiner, J. 2002, Ph.D. thesis, Univ. California, Berkeley

Wilson, R. W., Baldwin, J. E., Buscher, D. F., \& Warner, P. J. 1992 MNRAS, 257,369

Wing, R. F. 1997, J. AAVSO, 25, 63

Woitke, P., Sedlmayr, E., \& Lopez, B. 2000, A\&A, 358, 665

Ya'ari, A., \& Tuchman, Y. 1999, ApJ, 514, L35

Young, J. S., et al. 1999, in IAU Symp. 191, Asymptotic Giant Branch Stars, ed. T. Le Bertre, A. Lebre, \& C. Waelkens (San Francisco: ASP), 145 